\title{
Memory for numbers: Nominal vs. magnitude information
}

\author{
JAMES V. HINRICHS and LAURA R. NOVICK \\ University of Iowa, Iowa City, Iowa 52242
}

\begin{abstract}
Across three paired associate learning experiments, the recall of four-digit number responses to word stimuli favored the most significant digit over the least significant (magnitude encoding) rather than exhibiting typical bowed serial position functions (nominal encoding). Only with instructions emphasizing exact recall of each individual digit did recall functions revert to bowed curves. The results are interpreted as evidence for two kinds of number coding in semantic memory.
\end{abstract}

One of the best established facts of human learning and memory is the ubiquitous bowed serial position curve. Across a wide variety of materials, list lengths, scoring methods, and experimental manipulations, the shape of the serial position function remains remarkably constant (McCrary \& Hunter, 1953). Although the mechanism underlying the serial position function is still a controversial theoretical issue, there can be little doubt that it depends on the item-by-item processing of the to-be-remembered members of the sequence. Even when numbers are grouped by naming constraints (Bower \& Winzenz, 1969, see below), bowed retention functions occur both within groups and over the entire sequence. The consistency and pervasiveness of the serial position curve-in particular, the generally poorer retention of interior items of the list relative to end items-was the impetus for this investigation of alternative ways that numbers may be represented in memory.

Another perspective contributing to the present investigation was the observation that we usually think of numbers as magnitudes for which approximation of the exact value of the number is a reasonable and common mode of representation. The focus of the research reported here is on the two types of memorial representation implied by magnitude and nominal coding of numbers. In particular, as outlined below, the two interpretations predict different serial position functions and different levels of accuracy in remembering the values of numbers. The experiments were primarily concerned with identifying which kind of coding was used by subjects and the extent to which their representation could be altered by instructional manipulations.

This research was supported by National Institute of Education Grant G-0178 to J. V. Hinrichs. We thank Gayle Klouda and Janis Berie for their assistance. Requests for reprints should be sent to J. V. Hinrichs, Department of Psychology, University of Iowa, Iowa City, Iowa 52242.

\section{Nominal Representation: Bowed Serial Position Functions}

Most studies employing numeric stimuli only measure serial position effects over the entire list (e.g., Murdock, 1968, especially Experiment 6), but Bower and Winzenz (1969, Experiments 2 and 3) simultaneously examined serial position curves within and across items. Their stimuli consisted of lists of 12 digits that were read in groups of one- to four-digit numbers. For example, 13649 might be presented as "thirteen, six hundred forty-nine" or as "one, three (pause), six, four, nine." Subjects were then told to recall all 12 digits in their correct serial locations. In general, digits at the beginning and end of the list were recalled best, and digits from the middle of the list were less likely to be recalled, consistent with the typical serial position curve found by other investigators. However, Bower and Winzenz also found serial position curves within two- and three-digit subsequences. The results showed approximately flat or bowed serial position curves within each two- to threedigit group, as well as a bowed recall function across all 12 positions. Unfortunately, Bower and Winzenz did not present serial position curves for lists containing fourdigit numbers, for which it would be much easier to discern the shape of the serial position function than for shorter sequences; however, they do assure us that lists containing other patterns of groups showed similar results.

Similarly, Estes $(1972,1973)$ found flat or bowed serial position functions, depending on the length of the retention interval, for a series of four letters presented in rapid succession. Although his studies were primarily concerned with manipulating the auditory coding and rehearsal conditions for remembering four-letter sequences, his conclusion that the curvature of the serial position functions develops over the retention interval as the memory maintenance process fails would apply equally well to number sequences. Particularly noteworthy was the observation, consistent with other shortterm memory serial position results, that recall was rela- 
tively better in the later (recency) serial positions as the retention interval increased, especially when a categorizing task was imposed at the time of presentation.

\section{Magnitude Representation: Approximation of Numeric Values}

Although the Bower and Winzenz (1969) studies used different ways of coding numbers, all methods employed verbal labels and presented numbers as arbitrary items. An alternative and perhaps more common way of thinking about numbers is as magnitudes. We can quite easily determine that one car costs more than another or that one pile of blocks contains more blocks than a neighboring pile. Early schooling stresses the magnitude aspect of numbers through the practice of arithmetic operations. Recent studies have supported the idea that numbers are represented internally as magnitudes (Hinrichs, Berie, \& Mosell, in press; Hinrichs, Yurko, \& Hu, 1981; Moyer \& Landauer, 1967, 1973; Sekuler, Rubin, \& Armstrong, 1971).

Inherent in the interpretation of numbers as magnitudes is the idea of rounding or approximation. Thus, we may say that there are "about a dozen" donuts when there are actually 11 or 13 donuts, and we may say that a bicycle costs "about $\$ 100$ " when its exact price is $\$ 112$. In both cases, the approximations would give the individual considerable information about the value of the number. This magnitude property of numbers provides a strong contrast to the way in which we typically use numbers and other symbols as labels. An interesting property of numbers-and the focus of the present investigation-is that numbers can be used in two senses. Numbers can be used in a nominal sense as arbitrary labels (e.g., telephone and identification numbers). The ordinal properties of numbers are not essential for this usage; any set of symbols would do just as well. In the magnitude mode, however, it is the value of the numbers that is important. Here we used numbers as amounts, especially in the measurement of size, money, time, and so forth. Strictly speaking, the use of numeric symbols is not crucial, but the ordinal property of numbers must be preserved. [In speaking of magnitude, we are deliberately blurring the distinction between the cardinal and ordinal use of numbers as well as between particular scalar properties. In keeping with Stevens' (1951) interpretation, we are only distinguishing between nominal scales and the higher scales. We combine the more exact definitions of ordinal, interval, and ratio scales under the rubric of "magnitudes."]

\section{Comparison of Nominal and Magnitude Representation}

The particular distinction that is of concern to the present investigation is the fact that exact symbol identification is essential for the nominal use, whereas approximate values carry considerable information in the magnitude mode. Thus, to a certain extent it is useful to think of a car that costs $\$ 8,317$ as costing about $\$ 8,000$, but one cannot expect to reach John Doe on the phone by dialing $353-8000$ if his number is really $353-8317$. The question raised in the present investigation is whether these two different uses of numbers are represented differently in memory. If we are required to retain some numerical information, do we remember the numbers differently when they are used in a nominal sense as opposed to when they are used in a magnitude sense? It is proposed that serial position functions may be used to distinguish between the two types of representation.

At least one previous study suggests that some placevalue information is maintained when numbers are encoded into memory. Dale and Baddeley (1966) found that errors in remembering two-digit numbers were not random. Intrusions in free recall and the effectiveness of distractions in recognition tests were both more related to the value of the tens-place digit than the ones-place digit. The investigators did not speculate on the nature of the memorial representation of numbers and limited their study to two-digit numbers. The present study considered larger numbers and more extensive analyses of place-value effects.

Three experiments are reported that examine the encoding and retention of numeric information. The magnitude numbers used were described to subjects as four-digit car prices and the nominal numbers were described as the last four digits of telephone numbers. If people do indeed have different internal representations for these two types of numbers, two major results are expected. First, the mean absolute difference between the presented numbers and the subjects' remembered responses should be much smaller for those subjects who are told that the numbers are prices than for those subjects who are told that they are telephone numbers. Second, and partially the reason for the first expected result, the "price" subjects should tend to acquire and retain the thousands-place number best, then the hundreds-place number, the tens-place number, and, finally, the ones-place number. "Telephone" subjects, however, should show either no difference in retention over digit serial position or a serial position learning curve analogous to those found in typical serial recall studies. Alternatively, subjects may be strongly biased to represent numbers in one mode or the other regardless of instructional set. Four-digit numbers were used because they make sense for both prices and telephone numbers, and prior research (e.g., Bower \& Winzenz, 1969; Estes, 1972, 1973) suggests that they should contain enough positions to allow different serial position effects to be discerned.

\section{EXPERIMENT 1}

The first experiment was designed to determine the mode of representation, nominal or magnitude, of fourdigit numbers favored by instructions about the nature 
of the numbers to be remembered. Subjects were required to learn paired associate lists with four-digit numbers as responses. The encoding of the numbers was manipulated by the nature of the stimuli (common first names, automobile model names, or paralogs) and the instructions (consider the numbers to be prices or consider the numbers to be part of a telephone number). Paralogs (nonsense words) were tested to determine the effect of stimuli with no prior association with numbers except through instructions.

\section{Method}

Subjects. Subjects were 42 University of Iowa students who participated to partially fulfill course requirements. The data from three subjects were deleted from the analyses because those subjects failed to follow instructions. Final analyses were performed on data for 10 subjects in each of the car-price, nametelephone, and paralog price conditions, and for 9 subjects in the paralog-telephone condition.

Design and Procedure. Four 10-item word-number paired associate lists were constructed using a 2 by 2 factorial design with type of number and word-number relatedness as independent variables. For each list, the same set of four-digit numbers was used. However, half of the subjects were told that it might help them to learn the list if they thought of the numbers as the last four digits of a telephone number, and the others were told to think of the numbers as prices. The relatedness of the words to the numbers was also manipulated. In the related condition, five common male and five common female first names (Battig \& Montague, 1969) were used with the telephone number instructions and 10 recent car model names ("The 1979 Cars," 1979) were used with the price instructions. In the unrelated condition, the same set of 10 nonsense words (Noble, 1952) was used with both telephone and price instructions. Subjects were told that it might be helpful to think of the nonsense wordnumber pairs as either (1) people's names and telephone numbers or (2) car names and car prices. Thus, there were actually only three separate lists: one with first names, one with car names, and one with paralogs. The subjects were tested in small groups of three to five individuals.

During the study trials, the word and number of each pair were presented simultaneously on slides for $5 \mathrm{sec}$. Five separate orders of the stimuli were prepared for the study trials, and each order was presented twice for a total of 10 trials.

After each study trial, subjects were given a $2-$ min period in which they were to recall as many of the numbers as they could. Responses were made by writing the number on a line next to the word with which it was paired. In order to facilitate an analysis of digit-by-digit learning, subjects were asked to write down four digits if they remembered any digits at all. A separate sheet of paper was used for each test trial. Five separate orders of the stimulus items, which were different from the study orders, were prepared for the recall sheets, and each order was included twice in the test booklets. Each subject received a different random order of the 10 recall sheets.

Before learning the test list, subjects were given three trials of practice on a five-item list consisting of color word/CCC pairs. There were three different orders of the stimulus items for the study task and three additional orders for the recall booklets. The test sheets were randomly arranged in the recall booklets. Presentation of the practice list was identical to that of the test list. The recall period, however, lasted only $1 \mathrm{~min}$.

\section{Results and Discussion}

Performance on the following dependent variables was considered: (1) number of items attempted,(2) num- ber of items (four-digit numbers) correctly recalled, (3) number of digits correctly recalled regardless of serial position, (4) number of digits recalled in their correct serial positions, (5) number of digits correctly recalled regardless of serial position minus number of digits recalled in their correct serial positions, (6) mean absolute difference between the numbers presented and subjects' responses, and (7) number of digits correctly recalled by serial position. No task (prices vs. telephone numbers) or relatedness (car or first names vs. paralogs) effects were found for either the number of items attempted or the number of items correctly recalled. Collapsed across the 10 trials, about six of the eight items attempted on each trial were recalled correctly regardless of condition. Thus, neither the task nor the relatedness condition was harder than the other, permitting the remaining analyses.

The results can be easily summarized: There were no task differences for any of the dependent measures. Mean absolute differences were 414 and 615 for the price and telephone conditions, respectively $(p>.10)$. Serial position recall was also virtually identical for the two tasks: 28.7 and 29.5 digits correctly recalled, regardless of serial position, per trial for the telephone and price subjects, respectively $(\mathrm{F}<1)$. The telephone subjects also failed to remember more digits in their correct serial positions, despite the fact that knowledge of all four digits and their positions is necessary if one is to make a telephone call (means of 26.1 and 27.4 for the telephone number and car price conditions, respectively, $\mathrm{F}<1$ ). A less stringent test of the hypothesis would be to say that the difference between number of digits correctly recalled regardless of serial position and number of digits recalled in their correct serial positions should be larger for telephone than price subjects. Although this difference was in the expected direction (means of 2.6 and 2.1 , respectively), it also failed to reach significance $[F(1,35)=1.35, p>.25]$. Only trial number contributed significantly to the experimental variance, providing the uninteresting result that performance improved with practice.

Given that the experimental manipulation produced no significant differences, the remaining question of interest is whether the observed recall pattern was produced by nominal or magnitude coding. To provide the answer, performance was examined across the four serial positions within the numbers. As before, performance improved over trials, but trial did not interact with any other variable. Consistent with the lack of task differences described above, the Task by Serial Position interaction did not approach significance $(\mathrm{F}<1)$. The main effect of serial position $[F(3,105)=17.15, p<.01]$ showed that for both tasks, the numbers appeared to be learned in the order of the serial positions of the digits. The mean number of digits recalled (of 10 ) for the thousands, hundreds, tens, and ones positions were 6.91, $6.82,6.56$, and 6.46 , respectively. Although the differ- 
ences are small, the ordering was as predicted by magnitude encoding for both price and telephone number conditions. Most people would probably agree that telephone and identification numbers are in some sense different from prices and lengths. However, the first experiment failed to find differences in memory representation. In fact, much to our surprise, and contrary to the vast array of studies showing bowed serial position retention functions, both types of numbers showed memory performance decreasing in strict left-to-right order, consistent with magnitude encoding.

\section{EXPERIMENTS 2 AND 3}

Given the unexpected and counterintuitive results of Experiment 1, it seemed necessary to repeat the experiment with some improvements. Since subjects in Experiment 1 appeared to find the task fairly easy (70\% of the items were learned by Trial 6), Experiments 2 and 3 used a more demanding paired associate task to examine the encoding and retention of nominal and magnitude numbers. The stimulus list contained interleaving study and test trials, such that retention could be tested with zero to eight trials intervening between initial encoding and subsequent cued recall. Also, subjects were required to respond to every test item, unlike in Experiment 1, to prevent subjects from concentrating on just a few items. Finally, and most important, the wording of the instructions was manipulated across experiments. Experiment 2 instructions were similar to those used in Experiment 1, but the instructions in Experiment 3 emphasized exact recall in the telephone number condition and approximation ("closeness") in the car price condition.

\section{Method}

Subjects. Forty undergraduates (18 males and 22 females) at the University of Iowa participated in Experiment 2. The data from three subjects (one male and two females) were excluded from the analyses due to scoring difficulties. The subjects for Experiment 3 were 29 undergraduates from the same university. Again, the data from three subjects were excluded from the analyses due to scoring difficulties, and therefore the final analyses were performed on data from 19 males and 7 females. All subjects participated to partially fulfill introductory psychology course requirements.

Materials. Word-number paired associates were again used. Since two sets of 30 stimuli were needed, additional first names and car names were selected from the same sources used in Experiment 1 (Battig \& Montague, 1969; "The 1979 Cars," 1979). The first names consisted of the 20 most common female and the 20 most common male names, using the more common spelling pattern in any ambiguous cases. The car model names were selected to minimize confusion among the names. The fourdigit response numbers were chosen such that each of the 10 digits appeared equally often in each of the four serial positions. They were further constrained in that (1) the first two digits in any set of 30 numbers could not be the same and (2) no number contained repeated digits.

The stimuli were arranged in a continuous paired associate list with interleaving test and study trials. A word-number pair would be presented for study on one trial, and then some number of interpolated trials later would be tested. The number of inter- polated items was either zero, one, two, four, or eight study or test trials. A test list consisted of 69 presentations arranged to include 30 test trials, 30 first presentations of the test items, and 9 fillers. The 30 test trials were approximately distributed as six blocks, each containing one test at each of the five intervals. The response items were distributed to match the stimulus items, with particular numbers randomly assigned to the word stimuli. The number would be paired with the stimulus at the first occurrence of the stimulus and a question mark indicating a recall attempt would be paired with the second presentation of the stimulus. The name and automobile lists had the same order of presentation and testing.

For Experiment 2, the assignment of the number list to the stimulus word pairs was counterbalanced-Each number response list was assigned to the name stimulus list half of the time and to the car stimulus list the other half of the time. Thus, for example, half of the subjects who received the car prices task first saw the first list of numbers with the car names, and the other half saw the second list of numbers with the car names (and the first list of numbers with the first names). In Experiment 3, the number response lists were randomly assigned to the stimulus lists so that the same numbers were always paired with the car models and the first names.

Design and Procedure. All subjects first completed a wordword practice list to familiarize them with the testing procedure and the pacing of the task. Then each subject was tested on both stimulus list, half of the subjects receiving the name list first and half receiving the car list first. In addition, in Experiment 2 half of the subjects saw one set of response numbers first and the others saw the other set of numbers first. The subjects, tested in groups of four to eight individuals, were shown slides at a rate of $5 \mathrm{sec} / \mathrm{item}$.

Prior to the name list, subjects were given instructions describing the nature of the task and the requirement of associating a four-digit number with a familiar first name. They were instructed that "it may be easier for you to learn these items by thinking of the numbers as the last four digits of a telephone number where you already know the first three numbers." An example was given using the three-number exchange used in the University of Iowa residence halls and an arbitrary four-digit number. In Experiment 3, the following instructions were added: "It is important that you try to recall the four digits as accurately as possible. Your answers will be scored on the exactness of each number in the sequence." Before the automobile list, the subjects were instructed "to think of the numbers as prices of new and used automobiles that you might be interested in purchasing." They were given several examples incorporating familiar names of automobiles and prices. One example used a leading zero (e.g., Pinto-0962) to familiarize the subjects with the possibility of its occurrence on the list. The final instructions in Experiment 3 were: "Your answers will be scored on the basis of how close they are to the price of the car. Give your closest approximation." The subjects had a brief rest period between the two lists.

On test trials the subjects recorded their recalled responses on an answer sheet with exactly four spaces for each response. In Experiment 2, subjects were instructed to write down four different digits on each test trial. Although the same sequences were presented, the strict prohibition against recalling repeated digits was removed in Experiment 3. For both experiments, subjects were required to place one digit in each of the four spaces for each item, even if it meant guessing some or all of the digits.

\section{Results and Discussion}

Experiment 2. Results. The dependent variables for the analysis of variance were (1) number of digits recalled in their correct serial positions, (2) number of digits recalled regardless of serial position, (3) mean absolute 
difference between subjects' responses and the correct answers, and (4) number of digits correctly recalled by serial position. The independent variables were (1) task (car prices vs. telephone numbers), (2) task order (car prices first vs. telephone numbers first), (3) number list, and (4) lag (zero, one, two, four, and eight intervening items). The only marginal task effect was for number of digits recalled regardless of serial position, with slightly more digits correctly recalled for the telephone number task than for the car prices task ( 2.73 vs. 2.64$)$ $[F(1,254)=5.00, p<.03]$. The most consistent effect was the main effect of lag. As would be expected, for all measures performance decreased with increasing lag ( $\mathrm{p}<.01$ in all cases).

Finally, the serial position curves for the car price and telephone number tasks were examined. The Task by Serial Position interaction again failed to reach significance $(\mathrm{F}<1)$, and the main effect of serial position $[F(3,99)=30.14, p<.01]$ showed that performance was a decreasing function of digit significance, as in Experiment 1. Out of a maximum of 30 digits correct per position ( 5 lags $\times 6$ instances/lag), subjects recalled $16.54,14.65,13.42$, and 13.66 digits in the thousands, hundreds, tens, and ones positions, respectively. Thus, Experiment 2 replicated Experiment 1 in showing magnitude or left-to-right encoding of numbers. The only other significant effect was the Task Order by Serial Position by Task interaction $[F(3,99)=5.18, p<.01]$. Car prices were recalled better and the serial position curves were flatter on the first task; telephone numbers were recalled better and the curves had greater slope on the second task.

Discussion. Experiment 2 was designed to be a better test for differences between nominal and magnitude coding of numbers than Experiment 1. First, one might expect any differences between the car price and telephone number conditions to be more likely to show up in a more demanding paired associate task. Also, the introduction of interleaving study and test trials allowed for a more controlled examination of digit retention across increasing lags between initial stimulus presentation and later recall test. Third, subjects were required to respond with four different digits on every test trial even if that meant guessing some or all of the digits (no omissions were allowed). Finally, a within-subjects design was used rather than a between-subjects design as in Experiment 1.

Thus, the fact that Experiment 2 replicated the findings of Experiment 1 allows us to place more confidence in the results. The serial position curves strongly support the idea that numbers are encoded as magnitudes, regardless of whether the particular usage preserves the scalar property of numbers. This finding fits well with the previous work on number judgments (Hinrichs et al., in press; Moyer \& Landauer, 1967, 1973; Sekuler et al., 1971 ) but contrasts with previous results (e.g., Bower \& Winzenz, 1969) on serial recall of numbers.
Experiment 3 attempted to reconcile this difference using the paradigm of Experiment 2, with the following slight modifications: (1) The number lists were not counterbalanced across word lists, since the results of Experiment 2 were the same regardless of with which set of numbers the first names and car names were paired, and (2) subjects were required to write down four digits on each recall trial, but to simplify the instructions, the requirement of no repeated digits was dropped. More important, subjects were specifically told to recall all four digits of the telephone numbers as accurately as possible and to give their closest approximation for the car prices. We hypothesized that a crucial part of the Bower and Winzenz (1969) instructions that accounted for their results was that subjects were told that their recall score would be the number of digits written in their correct locations; recall of the underlying digit sequence rather than of the particular digit groupings presented was stressed.

Experiment 3. Results. The data were first analyzed for order effects, that is, did subjects perform differently on the second task after completing the first? The three dependent measures (number of items correctly recalled, number of digits correctly recalled by serial position, and mean absolute difference) were inconsistent in their answers to this question. For number of items correctly recalled, there appeared to be no order effects: The main effects of task (car prices vs. telephone numbers) and condition (car prices first vs. telephone numbers first) were not significant, and these factors also failed to interact with any other factors (all ps $>.1$ ). Thus, in terms of exact recall of the complete four-digit number, subjects performed equally well regardless of task or task order. The only significant result was the main effect of lag $[F(4,96)=123.91, p<.01]$, as number of items correctly recalled decreased with increasing lag from a mean of 4.1 items (of 6) at Lag 0 to .2 items at Lag 8. The biggest decrease was from $\operatorname{Lag} 0$ to $\operatorname{Lag} 1$, with only 1.7 items recalled at Lag 1.

The results of the analysis on number of digits correct by serial position also showed no evidence for any order effects. The order variable condition failed to reach significance either alone or in combination with any other variable(s) (all ps > .1). However, the pattern of digit retention differed consistently for the two tasks, regardless of the order in which the tasks were presented. This effect will be discussed more fully following the discussion of mean absolute difference.

Unlike the other two variables, mean absolute difference was affected by the order in which the two tasks were presented. The Task by Task Order interaction was significant $[F(1,24)=17.01, p<.01]$. The mean absolute difference for the car prices task was approximately the same, regardless of whether subjects recalled the prices as the first task (a mean absolute difference of 1,648 ) or a second task (a mean of 1,541). For the telephone number task, however, performance was 
distinctly different from performance on the car prices task when the telephone numbers were presented first (an absolute difference of 2,206 ) but was very similar to the prices task when it was presented second (an absolute difference of 1,642). Consistent with this description, the main effect of task was significant when the analysis only considered the task that subjects completed first $[F(1,24)=8.98, p<.01]$ but failed to reach significance when the analysis was performed on the second task completed $(\mathrm{F}<1)$. The effect of lag did not vary with task presentation order $(\mathrm{F}<1)$, although the main effect of lag was reliable $[F(4,96)=41.89, p<.01]$. Mean absolute difference increased monotonically from 713 at Lag 0 to 2,451 at $\operatorname{Lag} 8$. The greatest increase was between Lag 1 (a mean of 1,275) and Lag 2 (a mean of $2,157)$.

Absolute difference is, of course, affected most by the value of the digit in the thousands place. It appears that when subjects received the tasks in the order car prices, then telephone numbers, they carried over the strategy of placing special emphasis on the first digit (which is appropriate to the prices task) to the telephone number task (in which all digits should be equally important). If this is true, one might expect the three-way interaction between number of digits correctly recalled by serial position, task, and task order to be significant. It was not $[F(3,72)=2.06, p>.1]$. Although the thousands-place digit was better recalled when the telephone numbers task was presented second (comparable to performance on the car prices task regardless of presentation order) than when it was presented first, recall for the other digits also improved. Thus, the shape of the serial position curve for the telephone-number task did not change much as a function of presentation order.

Figure 1 shows the serial position functions for each task, collapsed across presentation order. As is evident from the graph, there is a main effect of serial position

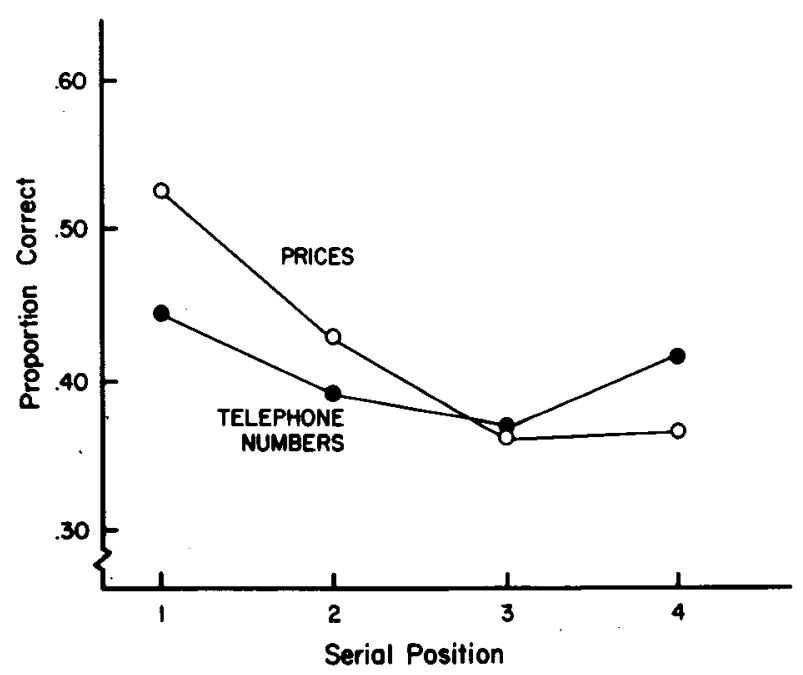

Figure 1. Proportion of digits correct by serial position for the car price and telephone number tasks in Experiment 3.

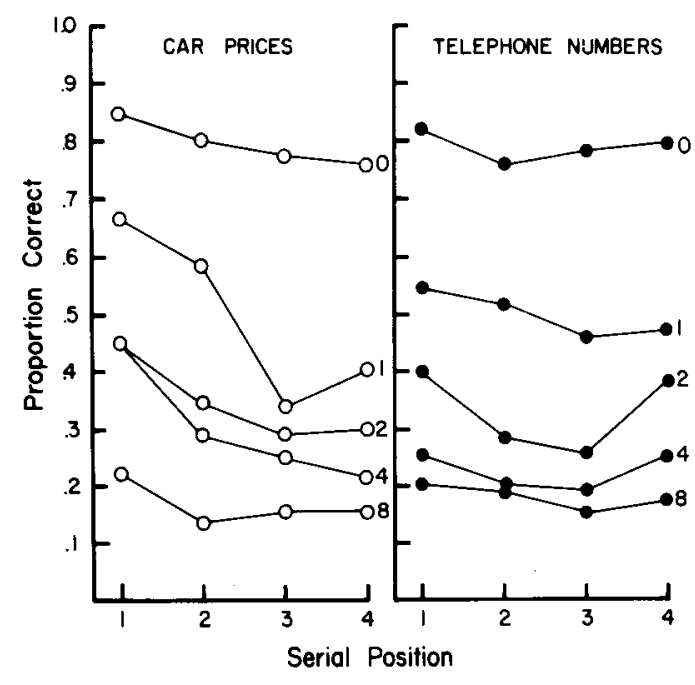

Figure 2. Proportion of digits correct by serial position and lag for the car price and telephone number tasks in Experiment 3. The parameter is the number of interpolated items between presentation and test.

$[\mathrm{F}(3,72)=28.31, \mathrm{p}<.01]$, as well as a significant Serial Position by Task interaction $[\mathrm{F}(3,72)=8.01, \mathrm{p}<.01]$. The serial position function for the telephone numbers is bowed, whereas the car prices curve is a decreasing function of serial position.

Not surprisingly, the number of digits correctly recalled per position decreased from 4.8 to 1.0 with increasing lag $[\mathrm{F}(4,96)=132.44, \mathrm{p}<.01]$. The Serial Position by $\mathrm{Lag}$ interaction was also significant $[F(12,288)=3.72, p<.01]$ : At Lag 0 , the digits in the four serial positions were approximately equally recalled (almost five digits per position). At Lag 1, information about the tens and ones positions was lost faster than information about the thousands and hundreds digits. At Lags 2 and 4, information about the hundreds-place number was lost faster than information concerning the thousands-place number. Finally, at $\operatorname{Lag} 8$, the curve was again fairly flat as only about one digit per position was recalled. Consistent with the notion of the scalar property of magnitude numbers, information about the last three digits (especially the tens- and ones-place digits) was lost faster in the car prices task than in the telephone numbers task. Similarly, information about the thousands-place digit was better retained in the car prices task. This Serial Position by Lag by Task interaction $[\mathrm{F}(12,288)=2.15, \mathrm{p}<.02]$ is illustrated in Figure 2. No other main effects or interactions were significant.

Discussion. Experiment 3 demonstrated that with the appropriate instruction set, subjects can encode magnitude (scalar) and nominal numbers differently. Specifically, subjects adopted either an approximation or a left-to-right encoding strategy for the car prices task, thus producing a serial position curve for number of digits recalled that was a decreasing function of serial 
position. For the telephone numbers task, when they attempted to remember all four digits exactly, the typical bowed serial position curve was found, very similar to Estes' (1973) results for four-letter strings (cf. Estes, 1973, Figure 3). Subjects' emphasis on the one or two most significant digits for the car prices was also evident in that those digits were retained the longest over increasing lag between initial encoding and subsequent test trial. In the telephone numbers task, however, information about the four digits was lost at a somewhat more constant rate as the serial position curves were fairly bowed at all lags.

\section{GENERAL DISCUSSION}

The present experiments examined whether nominal and scalar numbers are represented differently in memory. Such a difference might be expected, because approximate values carry considerable information in the magnitude usage of numbers, whereas exact symbol identification is necessary for nominal usage. In the paired associate task used here, encoding and memory differences consistent with the way in which the numbers are used would be expected to be evident in the following two ways: (1) The mean absolute difference between subjects' responses and the correct answers would be smaller for numbers presented as prices than for numbers presented as telephone numbers, and (2) memory for the telephone numbers would result in a flat or bowed serial position curve as a function of digit position, whereas memory for the prices would produce a serial position function that had recall decreasing monotonically from the thousands-place digit to the ones-place digit.

Before discussing any encoding differences as a function of task, it is important to note that the telephone number and car price tasks appeared to be equally difficult since there were no significant $(p<.01)$ task differences in the number of items correctly recalled for any of the experiments. Thus, any differences found on the other dependent measures are not likely to be an artifact of differential task difficulty.

Experiments 1 and 2 failed to provide any evidence that telephone numbers (nominal information) and car prices (magnitude information) are encoded or remembered differently. In fact, in both tasks the numbers appeared to be remembered in decreasing order of digit significance, consistent with magnitude encoding of the value of numbers. This result is surprising because virtually all previous investigations of number recall reveal conventional bowed serial position functions. In the absence of instructions to recall numbers digit-by-digit, and in some cases, even when exact retention was demanded, subjects exhibited a strong bias toward a magnitude representation, favoring recall of the thousands- and hundreds-place digit over the tens- and ones-place digit.

One possible interpretation of the results is that the evidence for magnitude encoding simply reflects a strong bias by subjects to use a left-to-right encoding strategy in the absence of explicit instructions to encode the digits in a particular order, perhaps induced by reading habits. There are several difficulties with this view. First, strict left-to-right processing is rare; bowed serial position effects predominate in most processing tasks from basic perception (e.g., Harcum, 1967) to letter recognition (e.g., Estes, 1972) to learning and memory (e.g., McCrary \& Hunter, 1953; Murdock, 1968). Second, attempts to require strict left-to-right ordering in recall of sequences generally fail. For example, Murdock's (1968) Experiment 6 revealed strong recency effects, even when subjects were told to recall digits in order from first to last (left to right), since credit would be given only up to the first error. Third, in the present study, the order of recall did not appear to differ across conditions or experiments. Although no systematic data are available, all subjects tended to report the four-digit sequences in the same left-to-right order, going back to make corrections as necessary. If left-to-right ordering habits are to be used to account for the results of Experiments 1 and 2, then some additional mechanism must be proposed to explain the failure to produce the same pattern for the telephone numbers in Experiment 3 . Consequently, magnitude encoding appears to be a simpler explanation of superior recall of initial digit positions.

Experiment 3 examined further the idea that subjects have a strong bias to think about numbers as magnitudes (i.e., special emphasis is placed on the scalar properties of numbers and the corresponding notion of place value) regardless of how the numbers are actually used. More specifically, we were interested in the possibility that this bias can only be overcome by explicitly instructing subjects to remember all four digits in the telephone numbers.

These hypotheses were supported. Experiment 3, in which subjects were instructed how to remember the numbers, was the only experiment to find strong differences between the two tasks for both the serial position curves and the mean absolute differences. However, these differences were most pronounced when the telephone numbers task and the car prices task were the first tasks completed. When subjects completed the telephone numbers task second, their performance on that task was strongly influenced by their having first completed the car prices task. In fact, when presented second, there was very little difference between the two tasks. Performance on the car prices task was not similarly influenced by initially completing the telephone numbers task.

The critical difference appears to be the item-by-item encoding usually demanded in experiments testing the recall of digit sequences as opposed to the whole number value encoding used by subjects in the present studies. The first we have called nominal encoding and the second, magnitude encoding. Although we found subjects strongly biased toward magnitude encoding in our experiments, a number of constraints and boundary con- 
ditions remain unexplored and may limit the generality of the results. In particular, the use of short sequences may be necessary; longer sequences are more likely to require sequential processing of individual numerals. Indeed, the general use of sequences beyond the usual memory span to examine serial position effects may explain why magnitude encoding is not observed more often. In any case, magnitude encoding of some numbers suggests flexibility in manipulating and remembering numbers other than strict digit-by-digit processing.

In general, the present results suggest that in remembering multiple-digit numbers, people tend to use a memory representation or encoding that incorporates the value or magnitude of the numbers. Support for this interpretation is provided by the finding that magnitude encoding predominates even when individuals are required to recall numbers presented as arbitrary labels (e.g., telephone numbers). These results are consistent with previous investigations measuring decision time and showing that numbers are compared like magnitudes (Hinrichs et al., in press; Moyer \& Landauer, 1967, 1973; Sekuler et al., 1971). However, we also found that when subjects were told to recall all of the presented digits and exact accuracy was stressed, performance reverted to the typical bowed serial position curve. Thus, when recall of individual symbols is emphasized, our results agree with those of previous investigators employing numbers and other stimuli. We interpret these results as evidence for two types of number encoding in semantic memory, with magnitude representation predominating and influencing performance even when nominal representation is assumed.

\section{REFERENCES}

Battig, W. F., \& Montaque, W. E. Category norms for verbal items in 56 categories: A replication and extension of the Connecticut category norms. Journal of Experimental Psychology Monograph, 1969, 80, 1-46.
Bower, G. H., \& Winzenz, D. Group structure, coding, and memory for digit series. Journal of Experimental Psychology Monograph, 1969, 80, 1-17.

Dale, H. C. A., \& Baddeley, A. D. Remembering a list of twodigit numbers. Quarterly Journal of Experimental Psychology, $1966,18,212-219$.

Estes, W. K. An associative basis for coding and organization in memory. In A. W. Melton \& E. Martin (Eds.), Coding processes in human memory. Washington, D.C: Winston, 1972.

Estes, W. K. Phonemic coding and rehearsal in short-term memory for letter strings. Journal of Verbal Learning and Verbal Behavior, 1973, 12, 360-372.

Harcum, E. R. Parallel functions of serial learning and tachistoscopic pattern recognition. Psychological Review, 1967, 74, 51-62.

Hinrichs, J. V., Berie, J. L., \& Mosell, M. K. Place information in multidigit number comparison. Memory \& Cognition, $1982,10,487-495$.

Hinrichs, J. V., Yurko, D. S., \& Hu, J. M. Two-digit number comparison: Use of place information. Journal of Experimental Psychology: Human Perception and Performance, 1981, 7, 890-901.

McCrary, J. W., \& Hunter, W. S. Serial position curves in verbal learning. Science, 1953, 117, 131-134.

Moyer, R. S., \& Landauer, T. K. Time required for judgments of numerical inequality. Nature, 1967, 215, 1519-1520.

Moyer, R. S., \& Landauer, T. K. Determinants of reaction time for digit inequality judgments. Bulletin of the Psychonomic Society, 1973, 1, 167-168.

Murdock, B. B., JR. Serial order effects in short-term memory. Journal of Experimental Psychology Monograph Supplement, $1968,76,1-15$.

The 1979 cars: Dealer cost vs. list price. Consumer Reports, 1979, 44, 208-209.

NoBle, C. E. An analysis of meaning. Psychological Review, $1952,59,421-430$.

Sexuler, R., Rubin, E., \& Armstrona, R. Processing numerical information: A choice time analysis. Journal of Experimental Psychology, 1971, 90, 75-80.

Stevens, S. S. Mathematics, measurement, and psychophysics. In S. S. Stevens (Ed.), Handbook of experimental psychology. New York: Wiley, 1951.

(Received for publication March 24, 1982; revision accepted July 21,1982 .) 